\title{
Enhancement of Flocculation and Dewaterability of MBR Activated Sludge Using a Hybrid System
}

\author{
Shiva A. Yousefi, Mustafa S. Nasser*, Abdelbaki Benamor, Reyad Shawabkeh ${ }^{2}$ \\ ${ }^{1}$ Gas Processing Center, College of Engineering, Qatar University, Doha, Qatar \\ ${ }^{2}$ Department of Chemical Engineering, University of Jordan, Jordan
}

\begin{abstract}
This study investigates the influence of hybrid coagulation-flocculation system on the flocculation and dewaterability efficiency of a real highly stable membrane bioreactor (MBR) sludge. Two organic coagulants, polyDADMAC (FL 4440) and polyamine (FL 2949) coupled with different structured polyacrylamides (PAMs) are used. Residual turbidity, zeta potential ( $\zeta$ ), flocs size and capillary suction time (CST) are used to assess the impact of the hybrid system on the degree of flocculation and dewaterability of MBR sludge. Addition of FL 4440 and FL 2949 prior to PAM has greatly reduced the turbidity and $\zeta$ of the sludge suspension by decreasing the required doses of PAMs $67 \%$ to $80 \%$ depending on the type of the PAMs used. Polyamine coagulant was more efficient in coagulation and hybrid coagulation-flocculation for MBR activated sludge. The impact of FL 2949 and FL 4440 was more significant on the linear structured PAMs with 71 and 80\% reduction in the required PAM's dose for 40\% CD (FO $4490 \mathrm{SSH}$ ) and 60\% CD (FO $4690 \mathrm{SSH}$ ), respectively followed by slightly and highly branched structured PAMs. Similar observations were observed for flocs size and dewaterability (CST) measurements of the flocculated MBR sludge. The results showed a maximum reduction of $58 \%$ in the flocs size for sludge conditioned with PAMs in the presence of FL 2949 and FL 4440. Nevertheless, comparable results were obtained for the dewaterability of the flocs produced by PAMs individually and hybrid system. Overall, this study successfully determines the effect of hybrid coagulation-flocculation on the flocculation performance of the MBR sludge.
\end{abstract}

Key words: Activated sludge; Membrane bioreactor; Polyelectrolyte; Polyacrylamide; Charge density; Volume reduction.

*Corresponding Author: Dr. Mustafa Nasser, Email: m.nasser@qu.edu.qa, Tel +974 4403-4380, Fax +974 44034371

\section{Introduction}

Activated sludge treatment is a major concern in wastewater treatment processes due to its complex nature and dynamic change in composition (Christensen et al., 2015; Vachoud et al., 2019). Sludge usually contains a large amount of water and has a hydrophilic property where it traps a large amount of water molecules within the sludge flocs (Liu et al., 2016; Pambou et al., 2016; Zhu et al., 2018). It contains small particles that form a stable suspension in water due to the electrostatic forces, making them difficult to settle and consolidate (Pambou et al., 2016; Reynolds and Richards, 1982). Hence, it is essential to condition the activated sludge prior to mechanical 
dewatering using chemical, physical or biological methods to reduce the sludge volume, transportation cost and ultimate sludge disposal, load on the subsequent unit operations such as filtration, and enhance sludge dewatering (Pambou et al., 2016).

In water treatment processes, one of the most well-known technologies for solid-liquid separation is coagulation/flocculation process (Kamaruddin et al., 2017). The aim of the coagulation process is to neutralize the negative charges of the stabilized colloidal particles where these destabilized particles aggregate and form micro flocs (Gregory and Barany, 2011; Kamaruddin et al., 2017; Oladoja, 2016). Different coagulants and flocculants such as aluminum sulfate, ferric chloride and polymers can be used for this purpose (Bolto and Gregory, 2007; Kamaruddin et al., 2017; "SNF Floerger," 2016).

In recent years, cationic polyelectrolytes such as polyacrylamide (PAM) are used as a flocculent and they showed a significant enhancement in the solid-liquid separation processes (Al-Dawery, 2017; Lau et al., 2017; Shifa M R Shaikh et al., 2017; Wei et al., 2018; Zhou et al., 2017). The flocs formed through charge neutralization and bridging mechanisms are sufficiently large and strong and can be separated by physical means like sedimentation, and they are highly resistant to any breakage due to the hydrolysis stress (Gregory and Barany, 2011; Oladoja, 2016). According to some previous studies, hybrid coagulation-flocculation has proved to be more effective in conditioning of activated sludge than when single coagulant or flocculant is used alone (Özacar and Şengil, 2000; Pambou et al., 2016; Zhao and Gao, 2016). In order to improve the quality of the produced sludge flocs (i.e. floc size, structure, strength and dewater-ability), a combination of coagulation and flocculation seems to be a good and simple method that need to be considered (Oladoja, 2016; Wei et al., 2018). Sludge conditioned with coagulants and flocculants as hybrid system is rarely reported; little is known about the application of the hybrid coagulationflocculation process for sludge conditioning and dewatering (Wang et al., 2018).

The presence of coagulant in tandem with flocculent helps with the formation of denser and stronger flocs which leads to faster settling and reduces the optimum dosage of the flocculant (Oladoja, 2016). Moreover, PAM can be partially hydrolyzed into toxic monomers such as acrylic acid and organic amine that lead to producing secondary pollution in sludge treatment process (ZHANG et al., 2007). Thus, it is essential to reduce its environmental impact by lowering the dosage through its combination with an appropriate coagulant (Aguilar et al., 2002).

Although, many studies have investigated the dewatering ability and performance efficiency of the hybrid systems, yet little is known in the literature on the influence of PAM's architecture, MW and $\mathrm{CD}$ on electro-kinetic properties and dewaterability of sludge when combined with strong or weak organic coagulants (Gregory and Barany, 2011). Combination of PAM with strong and weak organic coagulant such as polyDADMAC and polyamine, respectively is expected to have an influence on the electro-kinetics of flocs size and consequently the dewaterability of a real highly stable membrane bioreactor (MBR) sludge. Therefore, this study provides a comprehensive investigation of the synergistic influence of organic coagulants; polyDADMAC (FL 4440) and polyamine (FL 2949) combined with six different PAMs (with different architecture, MW and $\mathrm{CD}$ ) on the flocculation and dewatering enhancement of highly stable MBR sludge. This sludge has been extracted from an industrial MBR treating petroleum effluents. The study is focused on the impact of polymer structure and architecture on the electro-kinetic properties, particle size and consequently sludge dewaterability. 


\section{Material \& Methods}

\section{Material}

Sludge used in this study was collected periodically from the membrane tanks of a small (50 $\mathrm{m}^{3} /$ day capacity) MBR installation at a nearby petroleum industry site in Qatar. The collected samples were immediately transferred to the laboratory and stored at $-4^{\circ} \mathrm{C}$ for no more than four days prior to testing. The characteristics of the sludge sample were measured and the average values of 15-20 measurements are reported in Table 1. The six PAM flocculants and two organic coagulants tested (Table 2), supplied by SNF Floerger (France).

Table 1: The characteristics of MBR sludge used in this study

\begin{tabular}{l|cccc}
\hline Sludge property & Value & Min & Max & $\begin{array}{c}\text { Standard deviation } \\
\text { (SD) }\end{array}$ \\
\hline TSS, g/l & & & & 0.77 \\
VSS, g/l & 11.2 & 10.1 & 12.5 & 0.67 \\
SVI, ml/g & 10.1 & 9.13 & 11.3 & 3.6 \\
COD, mg/L & 89.9 & 84.3 & 94.9 & 3 \\
[NaCl], mg/L & 82 & 75 & 88 & 3.5 \\
Conductivity, $\boldsymbol{\mu S / c m}$ & 853 & 850 & 857 & 14.7 \\
pH & 1380 & 1362 & 1398 & 0.51 \\
Zeta potential, mV & 7.7 & 7 & 8.3 & 2.25 \\
Turbidity, NTU & -15.37 & -19.53 & -11.18 & 336 \\
D50, um & 2603 & 2048 & 2928 & 2.45 \\
CST, s & 17.12 & 13.42 & 21.77 & 1 \\
\hline
\end{tabular}

Table 2: PAM reagent characteristics

\begin{tabular}{|c|c|c|c|}
\hline Name & $\begin{array}{c}\text { Charge density } \\
\text { (\%) }\end{array}$ & $\begin{array}{l}\text { Molecular weight } \\
\quad\left(\text { g.mol }^{-1}\right)\end{array}$ & Structure \\
\hline FLOPAM FO 4490 SSH & $40 \%$ & 7-10 million & Linear \\
\hline FLOPAM FO $4690 \mathrm{SSH}$ & $60 \%$ & 7-10 million & Linear \\
\hline FLOPAM FO 4498 SSH & $40 \%$ & 7-10 million & Slightly Branched \\
\hline FLOPAM FO 4698 SSH & $60 \%$ & 7-10 million & Slightly Branched \\
\hline FLOPAM FO 4498 XXR & $40 \%$ & 4-7 million & Highly Branched \\
\hline FLOPAM FO 4698 XXR & $60 \%$ & 4-7 million & Highly Branched \\
\hline FLOQUAT FL 2949 (PolyAmine) & Very High & 60000 & \\
\hline FLOQUAT FL 4440 (PolyDADMAC) & Very High & 100000 & \\
\hline
\end{tabular}




\section{Methods}

PAM, polyDADMAC and polyamine stock solutions of concentration 2 g.L $\mathrm{L}^{-1}$ were prepared. Flocculation tests were conducted on the prepared sludge using a jar test apparatus (Stuart Flocculator SW6, UK) comprising six sets of 1L beakers stirred with standard rectangular paddles. Initially, coagulation test was performed by adding 5 to $70 \mathrm{mg} . \mathrm{L}^{-1}$ coagulants dosages to $250 \mathrm{ml}$ of sludge samples while mixing at 200 for 3 minutes and the optimum coagulants doses were obtained. For the hybrid coagulation-flocculation tests, the optimum doses of organic coagulants were added prior to PAMs addition. The samples containing coagulants were mixed at $200 \mathrm{rpm}$ for 2 mins, followed by further mixing with PAM of known dose for a period of 2 min at $180 \mathrm{rpm}$ $\left(G=350 \mathrm{~s}^{-1}\right)$, and subsequently the mixture was mixed for $20 \mathrm{~min}$ at low speed of $50 \mathrm{rpm}$. The resultant flocs were then allowed to settle for $15 \mathrm{~min}$ before extracting the supernatant at a depth of $50 \mathrm{ml}$ below sludge supernatant interface for turbidity (in NTU) and zeta potential $(\zeta, \mathrm{mV})$ analysis, using a Hach 2100N (US) turbidity meter and Zetasizer ZEN3600 (Malvern Instruments Ltd., UK) respectively. The flocculated sludge solids were taken for further analysis of floc size and bulk capillary suction time (CST) using Mastersizer 2000 (Malvern Instruments Ltd., UK) and Triton 319 Multi-purpose CST (Triton Electronics Limited, UK), respectively. Each measurement being replicated 3-5 times with a standard deviation (SD) of 0.02-4\%. Samples for $\zeta$ determination are pre-filtered to $0.45 \mu \mathrm{m}$.

\section{Results and Discussion}

\section{Optimum dose selection}

Figure 1 represents the trends in supernatant turbidity and zeta potential analysis of the MBR sludge samples flocculated with FL 4440 and/or FL 2949 coagulants. The optimum doses of these two coagulants were equated to the doses providing the maximum turbidity removal. Zeta potential has further supported the turbidity results demonstrating the reduction of the sludge's negative surface charge by 61 and 57\% for FL 4440 and FL 2949, respectively. Based on the same measurements i.e., zeta potential and turbidity the optimum dose of six PAMs were obtained which is summarized in Table 3 along with their corresponding values of turbidity, $\zeta, D_{50}$ and CST.

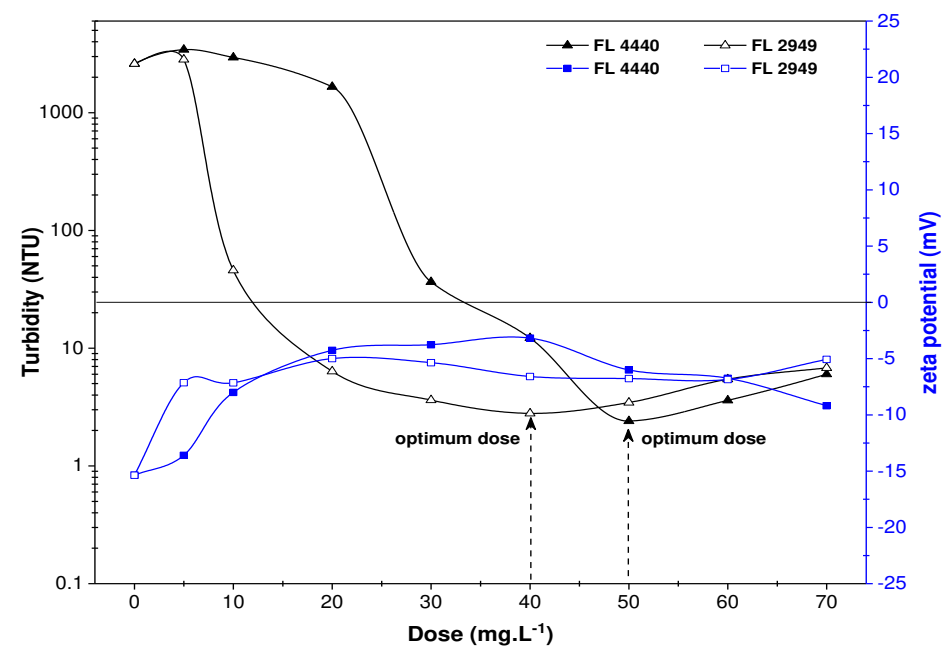

Figure 1: Variations in turbidity $(R S D=1-3 \%)$ and zeta potential $(\operatorname{RSD}=1-3 \%)$ for MBR sludge conditioned with FL 4440 and FL 2949 
Table 3: PAMs optimum doses along with measured parameters at their optimum doses

\begin{tabular}{|c|c|c|c|c|c|}
\hline flocculent & $\begin{array}{c}\text { optimum dose } \\
\left(\text { mg. } L^{-1}\right)\end{array}$ & $Z P(m V)$ & $\begin{array}{c}\text { Turbidity } \\
\text { (NTU) }\end{array}$ & $D_{50}(\mu m)$ & $\operatorname{CST}(s)$ \\
\hline $4490 \mathrm{SSH}$ & 70 & -4.86 & 2.0 & 245 & 5 \\
\hline RSD (\%) & & 1.05 & 2.8 & 2.3 & 2.1 \\
\hline $4690 \mathrm{SSH}$ & 50 & 5.22 & 1.57 & 241 & 7 \\
\hline RSD (\%) & & 1.24 & 2.6 & 3.6 & 1.8 \\
\hline $4498 \mathrm{SSH}$ & 30 & 5.6 & 0.47 & 155 & 8 \\
\hline RSD (\%) & & 3.3 & 4.2 & 2.5 & 1.5 \\
\hline $4698 \mathrm{SSH}$ & 30 & -2.95 & 1.80 & 155 & 7 \\
\hline RSD (\%) & & 1.2 & 1.8 & 3.5 & 1.2 \\
\hline 4498 XXR & 30 & -2.48 & 0.40 & 163 & 7 \\
\hline RSD (\%) & & 3.2 & 3.3 & 3.7 & 1.2 \\
\hline 4698 XXR & 10 & -5.5 & 0.9 & 69 & 6 \\
\hline RSD (\%) & & 2.4 & 2.5 & 2.34 & 1.3 \\
\hline
\end{tabular}

\section{Influence of polyDADMAC/polyamine combined with PAM}

Hybrid coagulation-flocculation of FL 2949 and FL 4440 was used to enhance the flocculation efficiency and achieve the required water clarity at the lowest possible doses of PAMs. The conditioning of MBR sludge was conducted in two stages: initially polyDADMAC and polyamine were added at their optimum doses followed by use of six different PAMs. Figure 2 shows an example of residual turbidity and $\zeta$ analyses of MBR sludge flocculated with FO $4490 \mathrm{SSH}$ coupled with FL 2949 (Figure 2a) and FL 4440 (Figure 2b). When linear PAM (FO 4490 SSH) is used alone, the highest turbidity removal is obtained at high PAM concentration of $70 \mathrm{mg} . \mathrm{L}^{-1}$ after which the system reaches nearly steady state condition. However, adding FL 4440 or FL 2949 at their optimum doses can significantly improve $\zeta$ and residual turbidity by reducing the initial dose of FO $4490 \mathrm{SSH}$ (linear PAM) to $20 \mathrm{mg} . \mathrm{L}^{-1}$. Therefore, for this case, $71 \%$ reduction in the initial optimum doses were achieved when combining it with $40 \mathrm{mg} . \mathrm{L}^{-1}$ of FL 2949 and $50 \mathrm{mg} . \mathrm{L}^{-1}$ of FL 4440. Conditioning of the sludge with the coagulant FL 2949 has reduced the required dose of FO $4490 \mathrm{SSH}$ from $\sim 80 \mathrm{mg} . \mathrm{L}^{-1}$ to $\sim 25 \mathrm{mg} . \mathrm{L}^{-1}$ and reached the PZC $(\zeta=0 \mathrm{mV})$.

Figure 3 illustrates the reduced optimum doses of PAMs obtained using hybrid system and the results are compared with the required doses for each PAM when used individually. In general, the results showed significant reductions in the optimal doses when hybrid coagulationflocculation is used in conditioning of the MBR sludge. These reductions range from 50 to $80 \%$ depending on the dose and type of the polymer. Chitikela and Dentel (Chitikela and Dentel, 1998) have also reported a $60 \%$ reduction of in the optimal doses by using hybrid coagulationflocculation system in conditioning and dewatering of anaerobically digested sludge. These reductions in the dose were more significant in the case of linear and slightly branched PAMs and less significant for highly branched PAMs.

The optimum doses were selected based on the maximum turbidity removal and $\zeta$ ranges -7 to +5 . The overall charges of colloidal particles and their stabilities are measured by $\zeta$, whereas turbidity is measured by water clarity and particle presence. Combining these two parameters are crucial for determining the degree of clarification, the effectiveness of agents and optimizing the efficiency and economy of the water treatment facility (Morfesis et al., 2009). On these bases, the highest reduction in PAM's initial optimum dose has been determined to be in the following order: FO 4490 SSH followed by FO 4690 SSH, FO 4498 SSH, FO 4698 SSH, FO 4498 XXR and FO 
4698 XXR. In terms of polymer CD, MW and molecular structure, the influence of CD and MW are superior to the effect of molecular structure.
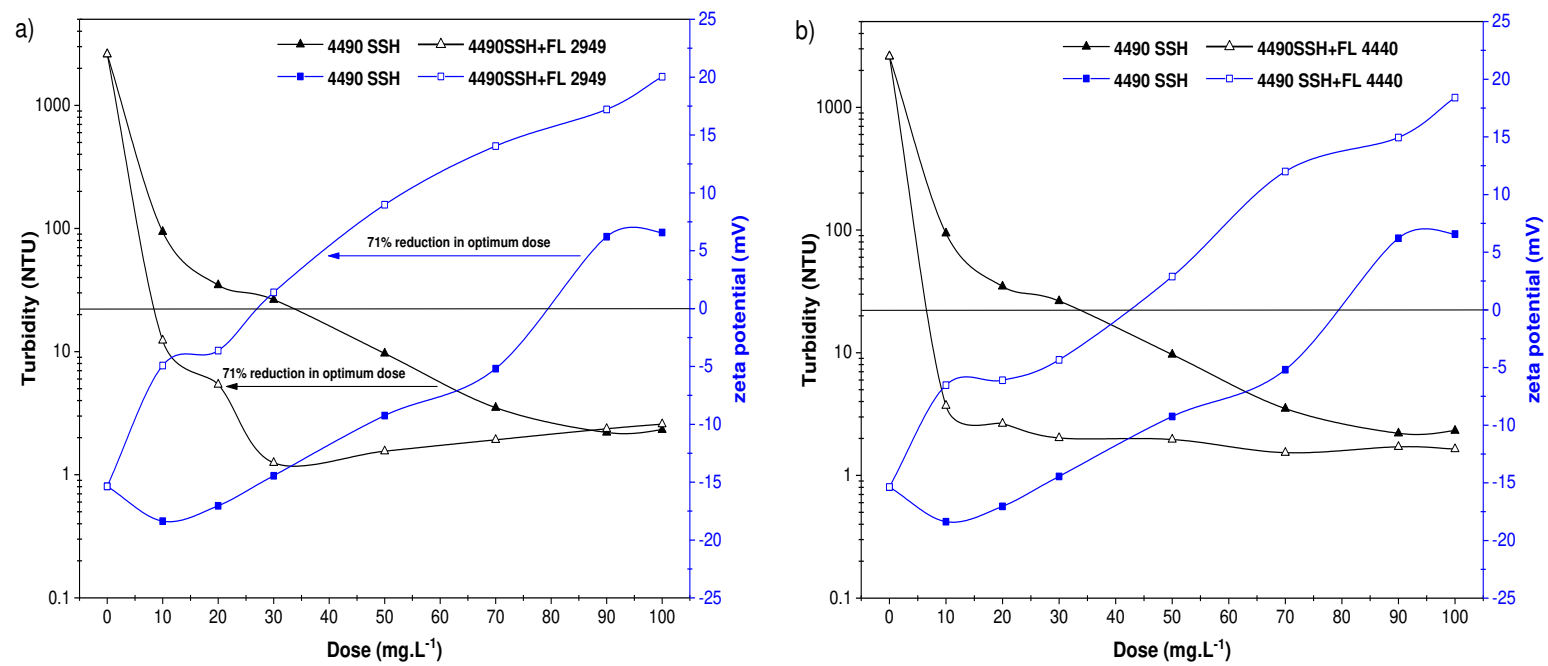

Figure 2: Turbidity $(\mathrm{RSD}=1-4 \%)$ and zeta potential $(\mathrm{RSD}=1-5 \%)$ trends for $\mathrm{MBR}$ sludge conditioned using FO 4490 SSH (linear PAM) combined with a) FL 2949 b) FL 4440

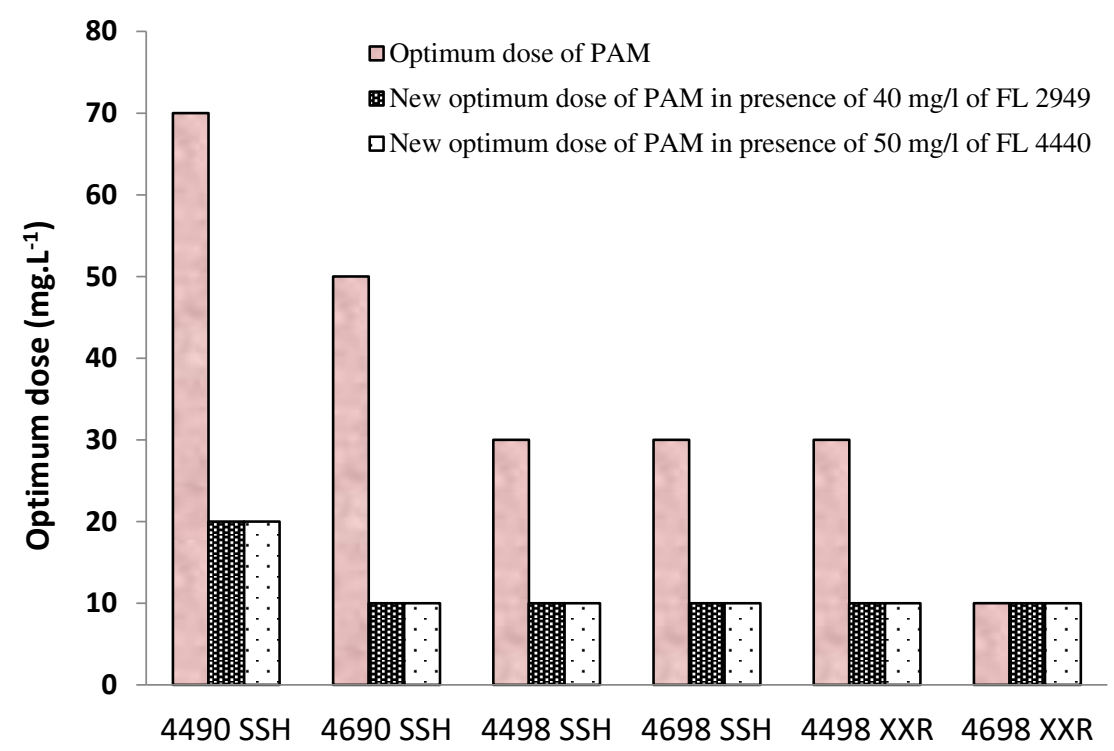

Figure 3: Optimum doses of MBR sludge conditioned with PAMs only and hybrid systems

\section{Effect of hybrid system on floc size analysis and CST of MBR sludge}

Floc size analysis was also conducted on the sludge conditioned with coagulants only and coagulants-flocculants hybrid system. As mentioned earlier, polyDADMAC and polyamine coagulants act as destabilizers and produce small flocs. By increasing the coagulant's dose, overall repulsive surface charges of the sludge colloidal particles have reduced through the compression 
of the electrical double layer. Consequently, the produced micro flocs sizes increase and the floc size distribution peak shifts to the right, representing a larger flocs formation. However, the flocs produced by polyamine and polyDADMAC were much smaller than those produced using different characteristics of PAM. On the other hand, $\mathrm{D}_{50}$ measurements were used to compare to flocs produced using FL 2949 and FL 4440 coagulants (Table 5). D 50 has increased from $17 \mu \mathrm{m}$ (unconditioned sludge) to $93 \mu \mathrm{m}$ for FL 2949 and $69 \mu \mathrm{m}$ for FL 4440 over the tested concentration range. This increase in floc size has reached to 74 and $67 \%$ at their optimum doses of FL 2949 and FL 4440, respectively.

The same analysis was performed for combined coagulation-flocculation system. In general, the flocs size has increased when either organic coagulants or PAMs are added to the MBR sludge having $\mathrm{D}_{50}=17 \mu \mathrm{m}$. However, when PAMs are used alone, larger flocs are produced compared to organic coagulants or hybrid systems where adsorption, charge neutralization and bridging mechanisms are involved.

Addition of coagulants, at their optimum doses coupled with PAMs, significantly enhances the adsorption of the amide group of PAM into the surface as particles get close to each other. Consequently, the bridging efficiency of PAM is improved and as a result, smaller and more compact flocs are formed in hybrid coagulation-flocculation system rather than when PAMs are used alone. According to the $\mathrm{D}_{50}$ results shown in Figure $4 \mathrm{a}$, comparing the $\mathrm{D}_{50}$ values of MBR sludge conditioned with PAMs and hybrid systems at their optimum doses, the influence of FL 2949 and FL 4440 on the linear structured PAMs is more significant followed by slightly and highly branched structured PAMs.

CST results have further supported the flocs size measurements. CST values were used to assess the effects of conditioning on sludge filterability or dewater-ability. Normally, larger flocs result in lower CST values compared to smaller flocs with narrow capillaries which do not release the water easily (Fitria et al., 2014; Turchiuli and Fargues, 2004).

However, the massive reduction in the flocs sizes does not increase the CST values significantly except when FO 4490 SSH (linear PAM) was used. In case of FO 4490 SSH (linear PAM), the reduction in the optimum dose from $70 \mathrm{mg} . \mathrm{L}^{-1}$ (when PAM is used individually) to $20 \mathrm{mg} . \mathrm{L}^{-1}$ (newly obtained dose for the hybrid system) has produced smaller flocs by $57 \%$. This could be the reason for the observed high CST differences in this case. However, this effect could be eliminated by slightly increasing the concentration of FO $4490 \mathrm{SSH}$ from $20 \mathrm{mg} . \mathrm{L}^{-1}$ to $30 \mathrm{mg} . \mathrm{L}^{-1}$. additionally; Figure 4b shows that when PAMs are combined with FL 2949, larger flocs and consequently lower CST were obtained compared to when PAMs are combined with FL 4440.

The results obtained for flocs size and CST clearly indicate that high reduction in the flocs size does not impact the dewater-ability of the produced flocs (i.e. CST) significantly. For example, when FO 4690 SSH was combined with FL 2949 and FL 4440, the flocs size has reduced by 63 and $65 \%$, respectively. However, CST is increased (i.e. dis-improved) by 5-7\% only (Figure 4b). Consequently, reducing the flocs size doesn't necessary significantly affect the dewaterability of the conditioned sludge and these minor effects can be eliminated by slightly increasing in the PAMs concentrations. 


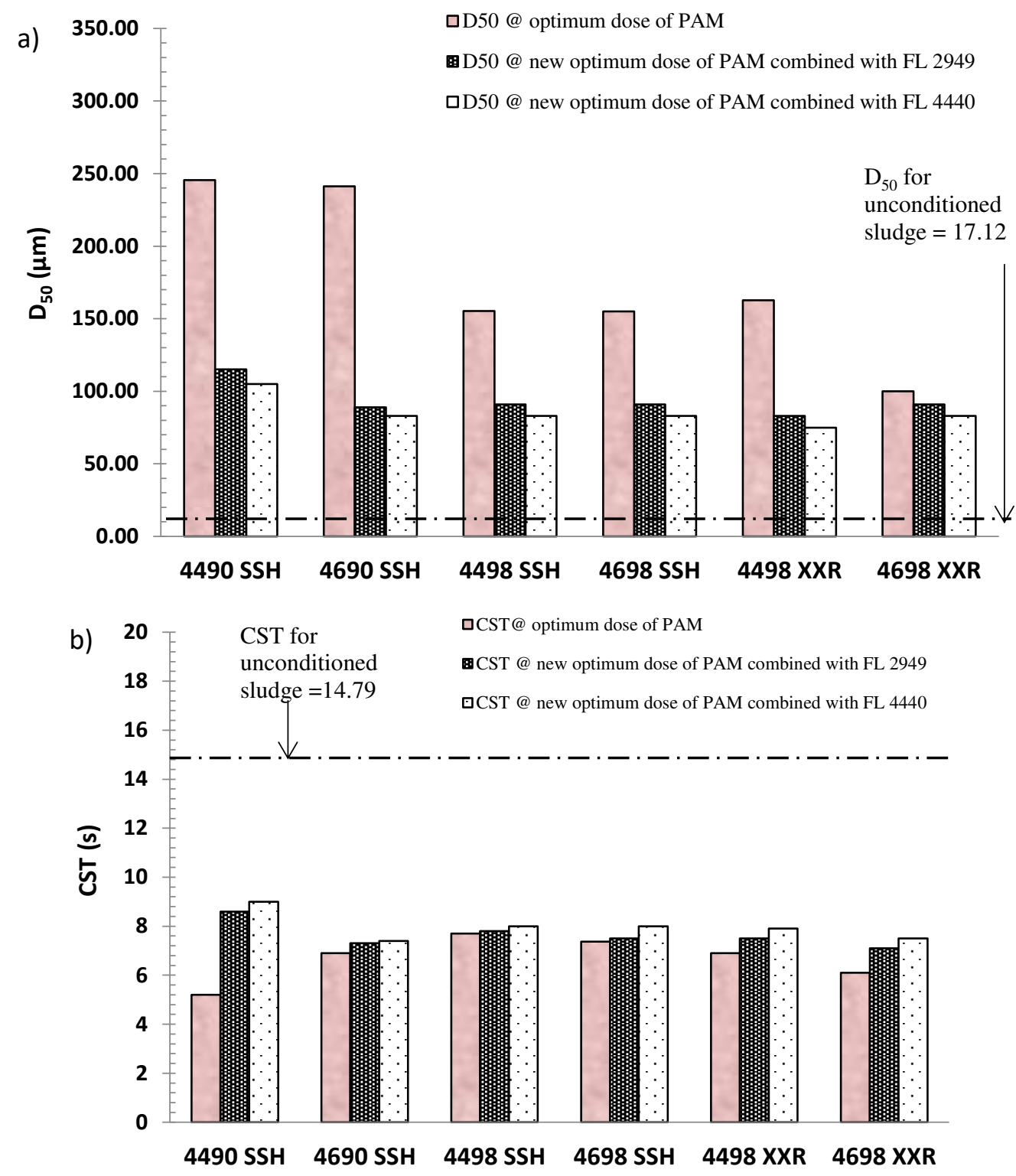

Figure 4: Variation in a) $D_{50}$ and b) CST for MBR sludge conditioned with PAMs and hybrid systems at their optimum doses

\section{Conclusion}

The main objective of this study was to optimize the flocculation performance of different polyacrylamides (PAM) by using hybrid coagulation-flocculation system in conditioning and dewatering of high stable membrane bio-reactor sludge.

The flocculation efficiency has improved for the hybrid system in terms of electro-kinetics and turbidity removal and amount of the required PAMs doses. This was significant in linear PAMs followed by slightly and highly branched structures. Minimal effect was observed for FO 4698 XXR (highly branched PAM) combined with two organic coagulants in terms of zeta potential and turbidity removal, since the required optimal conditions was achieved by using FO 4698 XXR independently. The flocs sizes in all cases have reduced significantly due to the 
mechanisms through which coagulation-flocculation take place. However, the pronounced reduction in the flocs size did not greatly distress the dewater-ability behavior which shows a great efficiency of hybrid coagulation-flocculation in conditioning of the highly stable MBR sludge.

\section{Acknowledgments}

The authors would like to acknowledge the support provided by Qatar University.

\section{References}

Aguilar, M.I., Sáez, J., Lloréns, M., Soler, A., Ortuño, J.F., 2002. Nutrient removal and sludge production in the coagulation-flocculation process. Water Res. 36, 2910-2919.

Al-Dawery, S.K., 2017. Degree of Flocculation and Interparticles Charges of Conditioned Municipal Activated Sludge using Mixed Polymers. J. Macromol. Sci. Part B 56, 578-594.

Bolto, B., Gregory, J., 2007. Organic polyelectrolytes in water treatment. Water Res. 41, 23012324.

Chitikela, S., Dentel, S.K., 1998. Dual-chemical conditioning and dewatering of anaerobically digested biosolids: laboratory evaluations. Water Environ. Res. 70, 1062-1069.

Christensen, M.L., Keiding, K., Nielsen, P.H., Jørgensen, M.K., 2015. Dewatering in biological wastewater treatment: A review. Water Res. 82, 14-24.

Fitria, D., Scholz, M., Swift, G.M., Hutchinson, S.M., 2014. Impact of sludge floc size and water composition on dewaterability. Chem. Eng. Technol. 37, 471-477.

Gregory, J., Barany, S., 2011. Adsorption and flocculation by polymers and polymer mixtures. Adv. Colloid Interface Sci. 169, 1-12.

Kamaruddin, M.A., Abdullah, M.M.A., Yusoff, M.S., Alrozi, R., Neculai, O., 2017. CoagulationFlocculation Process in Landfill Leachate Treatment: Focus on Coagulants and Coagulants Aid. \{IOP\} Conf. Ser. Mater. Sci. Eng. 209, 12083.

Lau, S.W., Sen, T.K., Chua, H.B., Ang, H.M., 2017. Conditioning of Synthetic Sludge and Anaerobically Digested Sludge Using Chitosan, Organic Polyelectrolytes and Inorganic Metal Cations to Enhance Sludge Dewaterability. Water, Air, Soil Pollut. 228, 358.

Liu, C., Lai, L., Yang, X., 2016. Sewage sludge conditioning by Fe(II)-activated persulphate oxidation combined with skeleton builders for enhancing dewaterability. Water Environ. J. 30, 96-101.

Morfesis, A., Jacobson, A.M., Frollini, R., Helgeson, M., Billica, J., Gertig, K.R., 2009. Role of Zeta $(\zeta)$ Potential in the Optimization of Water Treatment Facility Operations. Ind. Eng. Chem. Res. 48, 2305-2308.

Nasser, M.S., 2014. Characterization of floc size and effective floc density of industrial papermaking suspensions. Sep. Purif. Technol. 122, 495-505.

Nasser, M.S., James, A.E., 2008. Compressive and shear properties of flocculated kaolinitepolyacrylamide suspensions. Colloids Surfaces A Physicochem. Eng. Asp. 317, 211-221.

Nasser, M.S., James, A.E., 2006. The effect of polyacrylamide charge density and molecular weight on the flocculation and sedimentation behaviour of kaolinite suspensions. Sep. Purif. Technol. 52.

Oladoja, N.A., 2016. Advances in the quest for substitute for synthetic organic polyelectrolytes as coagulant aid in water and wastewater treatment operations. Sustain. Chem. Pharm. 3, 47- 
58.

Özacar, M., Şengil, I.A., 2000. Effectiveness of tannins obtained from valonia as a coagulant aid for dewatering of sludge. Water Res. 34, 1407-1412.

Pambou, Y.B., Fraikin, L., Salmon, T., Crine, M., Léonard, A., 2016. Enhanced sludge dewatering and drying comparison of two linear polyelectrolytes co-conditioning with polyaluminum chloride. Desalin. Water Treat. 57, 27989-28006.

Reynolds, T.D., Richards, P.A., 1982. Unit operation and process in environmental engineering. Wadsorth, CA.

Shaikh, S.M.R., Nasser, M.S., Hussein, I., Benamor, A., Onaizi, S.A., Qiblawey, H., 2017. Influence of polyelectrolytes and other polymer complexes on the flocculation and rheological behaviors of clay minerals: A comprehensive review. Sep. Purif. Technol. 187.

Shaikh, Shifa M R, Nasser, M.S., Hussein, I.A., Benamor, A., 2017. Investigation of the effect of polyelectrolyte structure and type on the electrokinetics and flocculation behavior of bentonite dispersions. Chem. Eng. J. 311, 265-276.

Shaikh, S.M.R., Nasser, M.S., Magzoub, M., Benamor, A., Hussein, I.A., El-Naas, M.H., Qiblawey, H., 2018. Effect of electrolytes on electrokinetics and flocculation behavior of bentonite-polyacrylamide dispersions. Appl. Clay Sci. 158, 46-54.

SNF Floerger [WWW Document], 2016. URL https://www.snf.co.uk/chemical-coagulants-usedwater-treatment/

Turchiuli, C., Fargues, C., 2004. Influence of structural properties of alum and ferric flocs on sludge dewaterability. Chem. Eng. J. 103, 123-131.

Vachoud, L., Ruiz, E., Delalonde, M., Wisniewski, C., 2019. How the nature of the compounds present in solid and liquid compartments of activated sludge impact its rheological characteristics. Environ. Technol. 40, 60-71.

Wang, H.F., Hu, H., Wang, H.J., Zeng, R.J., 2018. Impact of dosing order of the coagulant and flocculant on sludge dewatering performance during the conditioning process. Sci. Total Environ. 643, 1065-1073.

Wei, H., Gao, B., Ren, J., Li, A., Yang, H., 2018. Coagulation/flocculation in dewatering of sludge: A review. Water Res. 143, 608-631.

ZHANG, Z., LIN, B., XIA, S., WANG, X., YANG, A., 2007. Production and application of a novel bioflocculant by multiple-microorganism consortia using brewery wastewater as carbon source. J. Environ. Sci. 19, 667-673.

Zhao, S., Gao, B., 2016. Application of Enteromorpha as a new kind of coagulant aid in municipal sludge treatment. Desalin. Water Treat. 57, 7988-7998.

Zhou, Y., Zheng, H., Gao, B., Gu, Y., Li, X., Liu, B., Jiménez, A.M., 2017. Waste activated sludge (WAS) dewatering properties of an original hydrophobically modified polyacrylamide containing a cationic microblock structure. RSC Adv. 7, 28733-28745.

Zhu, C., Zhang, P., Wang, H., Ye, J., 2018. Ultrasonics - Sonochemistry Conditioning of sewage sludge via combined ultrasonication- fl occulation- skeleton building to improve sludge dewaterability. Ultrason. - Sonochemistry 40, 353-360. 